\title{
Optical properties of InAIGaAs quantum wells: Influence of segregation and band bowing
}

Jensen, Jacob Riis; Hvam, Jørn Märcher; Langbein, Wolfgang

Published in:

Journal of Applied Physics

Link to article, DOI:

10.1063/1.371096

Publication date:

1999

Document Version

Publisher's PDF, also known as Version of record

Link back to DTU Orbit

Citation (APA):

Jensen, J. R., Hvam, J. M., \& Langbein, W. (1999). Optical properties of InAIGaAs quantum wells: Influence of segregation and band bowing. Journal of Applied Physics, 86(5), 2584-2589. https://doi.org/10.1063/1.371096

\section{General rights}

Copyright and moral rights for the publications made accessible in the public portal are retained by the authors and/or other copyright owners and it is a condition of accessing publications that users recognise and abide by the legal requirements associated with these rights.

- Users may download and print one copy of any publication from the public portal for the purpose of private study or research.

- You may not further distribute the material or use it for any profit-making activity or commercial gain

- You may freely distribute the URL identifying the publication in the public portal 


\title{
Optical properties of InAIGaAs quantum wells: Influence of segregation and band bowing
}

\author{
J. R. Jensen a) and J. M. Hvam \\ Mikroelektronik Centret, The Technical University of Denmark, Building 345 East, \\ DK-2800 Lyngby, Denmark \\ W. Langbein \\ Experimentelle Physik EIIb, Universität Dortmund, Otto-Hahn Str.4, 44221 Dortmund, Germany
}

(Received 16 March 1999; accepted for publication 19 May 1999)

\begin{abstract}
Knowledge of the quaternary InAlGaAs material system is very limited for the composition range relevant for growth on GaAs substrates. We report on the characterization and modeling of InAlGaAs quantum wells with AlGaAs barriers, grown pseudomorphically on a GaAs substrate with molecular beam epitaxy. The quantum wells are characterized with photoluminescence, and the measured transition energies are modeled taking into account the influence of In segregation on the shape of the well potential. From the modeling we deduce a relation for the low temperature band gap of unstrained $\operatorname{In}_{x}\left(\mathrm{Al}_{y} \mathrm{Ga}_{1-y}\right)_{1-x} \mathrm{As}$, for $0 \leqslant x, y \leqslant 0.20$. The measured linewidths of the luminescence peaks are in agreement with the broadening expected from random alloy fluctuations and well width fluctuations with an effective interface roughness of 1.1 ML. (C) 1999 American Institute of Physics. [S0021-8979(99)00617-9]
\end{abstract}

\section{INTRODUCTION}

The ability to fabricate ternary and quaternary alloys of III-V semiconductors is of fundamental importance for applications in electronics and optoelectronics. Varying the alloy composition is the handle used to optimize the band gap, the effective masses, or the strain in a heterostructure layer.

Previously, different composition regions of the InAlGaAs material system have been studied in great detail. Especially the ternary subsystems of AlGaAs and InGaAs grown on GaAs are well characterized, due to their applications for devices in the wavelength range of 750-1000 nm. Also, band gap relations and effective masses for the quaternary InAlGaAs alloys, lattice matched to InP substrates, have been investigated, ${ }^{1-8}$ since here the band gap range covers the important wavelengths around $1.55 \mu \mathrm{m}$ for optical communication. However, little has been reported for InAlGaAs grown on GaAs substrates. This material system is important for low threshold multiple quantum well lasers at wavelengths shorter than $860 \mathrm{~nm},{ }^{9,10}$ and for growth of certain self-organized quantum dot structures. ${ }^{11}$ We also believe that the large flexibility of this system with respect to band gap and strain will prove useful for future designs of lowdimensional structures, e.g., realized by growth on cleaved edges.

For the properties of InAlGaAs on GaAs, two effects are especially important. First, due to the 7\% lattice mismatch between InAs and AlGaAs, it is only possible to grow a few nanometers of pseudomorphic material. For a given In content there is a critical thickness above which misfit dislocations are formed in the InAlGaAs layer, and since AlAs has almost the same lattice constant as GaAs the critical thickness is expected to depend on the In mole fraction as in

${ }^{\text {a)} E l e c t r o n i c ~ m a i l: ~ j r j @ c o m . d t u . d k ~}$
InGaAs. ${ }^{12,13}$ For the relevant In contents from 0.05 to 0.20 , the critical thickness ranges from several tens of nanometers to approximately $10 \mathrm{~nm}$, which means that quantum confinement effects are inevitable in pseudomorphic InAlGaAs layers, except for the lowest In mole fractions. Second, it has been shown that In tends to surface segregate during growth, ${ }^{14}$ smearing out the nominally sharp interfaces between a quantum well and its barriers. This leads to a blueshift of the transition energies compared to a perfectly square quantum well. ${ }^{15}$ Therefore, in order to perform a physically meaningful analysis of InAlGaAs quantum well transition energies and deduce a band gap relation for the bulk material, it is necessary to prepare samples in a way that allows the effects of segregation and quantum confinement to be determined independently.

\section{GROWTH OF STRUCTURES}

The measurements described in the following were made on three samples, referred to as S1, S2, and S3, grown by molecular beam epitaxy (MBE) on undoped (100) GaAs substrates. S1 contains eight $\operatorname{In}_{x} \mathrm{Ga}_{1-x} \mathrm{As} / \mathrm{GaAs}$ quantum wells of different nominal thicknesses and with a constant nominal In mole fraction of 0.10. S2 contains six $\mathrm{In}_{x} \mathrm{Ga}_{1-x} \mathrm{As} / \mathrm{GaAs}$ quantum wells with a constant thickness of $5 \mathrm{~nm}$, and $\mathrm{In}$ mole fractions varying from 0.05 to 0.30 . Finally, S3 contains five $\operatorname{In}_{x}\left(\mathrm{Al}_{0.17} \mathrm{Ga}_{0.83}\right)_{1-x} \mathrm{As} / \mathrm{Al}_{0.17} \mathrm{Ga}_{0.83} \mathrm{As}$ quantum wells with constant thicknesses of $8 \mathrm{~nm}$ and In mole fractions varying from 0.05 to 0.25 . The ratio of the $\mathrm{Al}$ to $\mathrm{Ga}$ mole fraction was kept constant during the growth of S3. In the following the five quantum wells in S3 will be referred to as QW1-QW5, QW1 being the quantum well with the lowest In content.

The growth rates were $0.7 \mu \mathrm{m} / \mathrm{h}$ for $\mathrm{GaAs}$ and 0.15 $\mu \mathrm{m} / \mathrm{h}$ for AlAs with a standard V/III flux ratio of approxi- 
mately 10 . Due to the large lattice mismatch between InAs and GaAs, reflection high energy electron diffraction (RHEED) oscillations cannot be obtained by growing InAs on a GaAs substrate. Instead, the InAs growth rate can be measured by subtracting the growth rate of GaAs from the growth rate of $\operatorname{In}_{x} \mathrm{Ga}_{1-x}$ As for low $x$ values. However, it was found that only an approximate calibration is possible in this way, since at low $x$ values $(x<0.05)$ the uncertainty on the InAs growth rate is large compared to the growth rate itself, and at high $x$ values $(x>0.15)$ the RHEED oscillations are strongly damped. We stress that the thicknesses and mole fractions stated above for S1, S2, and S3 are the nominal values obtained from the RHEED calibration, but as described in the following a better calibration of the In source was found using the photoluminescence (PL) peak positions of S2. All three samples were grown at $T_{\text {sub }}=530^{\circ} \mathrm{C}$ during the same loading of the MBE system, and prior to each growth the GaAs and AlAs growth rates were carefully measured using RHEED oscillations.

\section{MODELING OF SURFACE SEGREGATION AND TRANSITION ENERGIES}

For the growth of III-V arsenide heterostructures, In surface segregation has been observed in both InGaAs and InAlAs, the tendency being strongest in InAlAs. ${ }^{14,16,17} \mathrm{Ga}$ also segregates in AlGaAs, but the effect is weaker than in any of the In systems. Surface segregation can be modeled as a chemical reaction interchanging a group III atom in the surface atomic layer $(s)$ with a group III atom in the layer underneath $(b)$ :

$$
\begin{aligned}
& \mathrm{Ga}(b)+\mathrm{Al}(s) \rightarrow \mathrm{Ga}(s)+\mathrm{Al}(b), \\
& \mathrm{In}(b)+\mathrm{Ga}(s) \rightarrow \operatorname{In}(s)+\mathrm{Ga}(b), \\
& \mathrm{In}(b)+\mathrm{Al}(s) \rightarrow \operatorname{In}(s)+\mathrm{Al}(b) .
\end{aligned}
$$

The energies gained in each of the reactions have been deduced from previous measurements ${ }^{16,17}$ and consists of two terms, one corresponding to the change of chemical energy, $E_{s}$, and one corresponding to the change of elastic energy due to strain in the layers. ${ }^{14}$

To calculate the composition profile of the quantum wells investigated here, the growth was modeled in steps of one atomic layer. For each step the initial composition of the surface layer was calculated from the flux rates of the group III sources, and using the law of mass action the composition in thermal equilibrium with the layer underneath was found. For the segregation profiles of S1 and S2 only the equilibrium of Eq. (2) had to be considered, whereas for S3 the equilibrium of all three reactions was found restricting the solution to the one where the sum of group III mole fractions is 1 in the $(s)$ and $(b)$ layers, respectively.

From the composition profiles the potentials and effective masses in the quantum wells were calculated, taking into account the strain effects on the bandstructure. ${ }^{18}$ The energies of the electron and hole states were found by solving the Schrödinger equation, using an isotropic conduction band
TABLE I. Values of the physical parameters used for calculating the potentials and effective masses of the electrons and holes.

\begin{tabular}{lccc}
\hline \hline \multicolumn{1}{c}{ Parameter } & GaAs & AlAs & InAs \\
\hline Lattice constant, $\mathrm{d}(\AA)$ & $\left.5.6503^{\mathrm{b}}\right)$ & $5.6611^{\mathrm{b}}$ & $6.0583^{\mathrm{b}}$ \\
Stiffness constant, $C_{11}\left(\times 10^{10} \mathrm{~Pa}\right)$ & $11.88^{\mathrm{a}}$ & $12.02^{\mathrm{a}}$ & $8.329^{\mathrm{b}}$ \\
Stiffness constant, $C_{12}\left(\times 10^{10} \mathrm{~Pa}\right)$ & $5.38^{\mathrm{a}}$ & $5.70^{\mathrm{a}}$ & $4.526^{\mathrm{b}}$ \\
Stiffness constant, $C_{44}\left(\times 10^{10} \mathrm{~Pa}\right)$ & $5.94^{\mathrm{a}}$ & $5.89^{\mathrm{a}}$ & $3.959^{\mathrm{b}}$ \\
Relative electron mass, $m_{e} / m_{0}$ & $0.0667^{\mathrm{a}}$ & $0.15^{\mathrm{a}}$ & $0.0248^{\mathrm{c}}$ \\
Luttinger parameter, $\gamma_{1}$ & $7.1^{\mathrm{a}}$ & $3.76^{\mathrm{a}}$ & $19.7^{\mathrm{b}}$ \\
Luttinger parameter, $\gamma_{2}$ & $2.02^{\mathrm{a}}$ & $0.9^{\mathrm{a}}$ & $8.37^{\mathrm{b}}$ \\
Luttinger parameter, $\gamma_{3}$ & $2.91^{\mathrm{a}}$ & $1.42^{\mathrm{a}}$ & $9.29^{\mathrm{b}}$ \\
Hydrostatic deformation potential, $a_{g}(\mathrm{eV})$ & $-8.233^{\mathrm{c}}$ & $-8.110^{\mathrm{d}}$ & $-6.080^{\mathrm{c}}$ \\
Shear deformation potential, $b_{\mathrm{v}}(\mathrm{eV})$ & $-1.824^{\mathrm{c}}$ & $-1.7^{\mathrm{d}}$ & $-1.8^{\mathrm{b}}$ \\
Spin-orbit splitting, $V_{\text {so }}(\mathrm{meV})$ & $340^{\mathrm{b}}$ & $275^{\mathrm{b}}$ & $380^{\mathrm{b}}$ \\
\hline \hline
\end{tabular}

${ }^{\mathrm{a}}$ Reference 21 .

${ }^{\mathrm{b}}$ Reference 22.

${ }^{\mathrm{c}}$ Reference 23.

${ }^{\mathrm{d}}$ Reference 24

model and a six-band $k \cdot p$ model for the valence band. For unstrained $\mathrm{In}_{x} \mathrm{Ga}_{1-x}$ As the following relation was used for the low temperature band gap in $\mathrm{eV}:{ }^{19}$

$$
1.519-1.584 x+0.475 x^{2}
$$

and for $\mathrm{Al}_{y} \mathrm{Ga}_{1-y} \mathrm{As}$ in $\mathrm{eV}:{ }^{20}$

$$
1.519+1.36 y+0.22 y^{2} \text {. }
$$

The values of the other physical parameters used here for the binary materials are given in Table $\mathrm{I}^{21-24}$ For the ternary and quaternary materials, the values have been found by linear interpolation, however for the electron masses the inverse values have been interpolated. The conduction band offset was set to $67 \%$ of the total band offset, and the conduction band deformation potential was set to $90 \%$ of $a_{g} \cdot{ }^{18}$ To account for Coulomb interactions a well-width dependent exciton binding energy was used for the InGaAs structures in S1 and S2. ${ }^{19}$ Based on the binding energy for a heavy-hole exciton in an $8 \mathrm{~nm}$ InGaAs quantum well, the binding energies of the heavy- and light-hole excitons in S3 were taken as 7 and $8 \mathrm{meV}$. Since the contributions of the exciton binding energies to the transition energies calculated in the following are small, discrepancies of a few meV compared to the real values will not affect the results.

\section{TRANSITION ENERGIES}

In Fig. 1 the photoluminescence (PL) spectrum of S1 at $10 \mathrm{~K}$ is shown, and the positions of the peaks are compared to the calculated transition energies with and without segregation. First, the In content of the wells was fitted to $x$ $=0.092$, using a square well potential for the thickest well where the effect of surface segregation on the transition energy is negligible. Then a chemical energy of $0.34 \mathrm{eV}$ for In segregation in InGaAs was found, yielding the best overall fit to the transition energies. This is about a factor of two larger than the value obtained by Gerard et al., ${ }^{16,17}$ but as pointed out by Grandjean et al. ${ }^{25}$ the chemical energy itself depends on the temperature and can only be used as a fitting parameter. The tail of the segregation profile calculated for $E_{s}=0.34 \mathrm{eV}$ shows an exponential decay length of $2.7 \mathrm{~nm}$, 


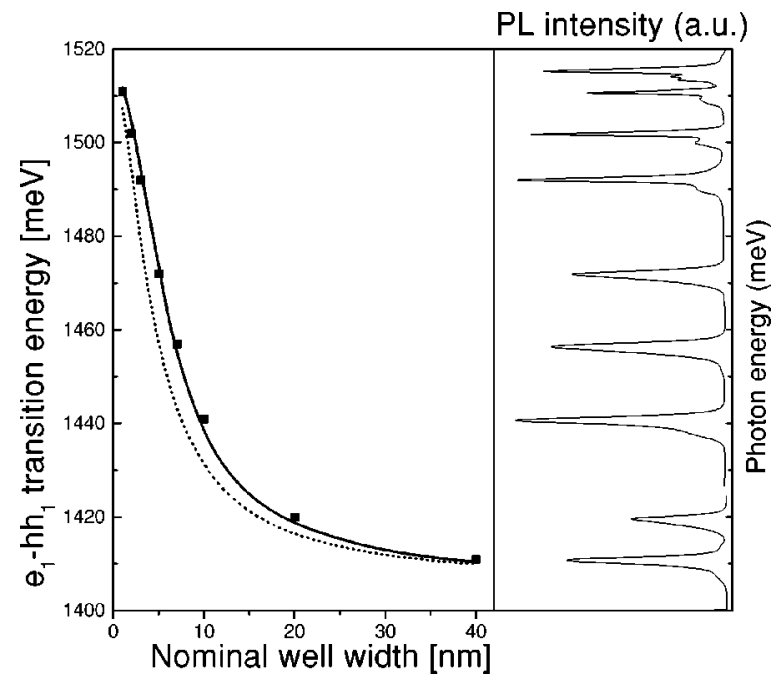

FIG. 1. Right: Photoluminescence spectrum of S1 at $T=10 \mathrm{~K}$. Left: Comparison of measured transition energies (squares) vs the nominal well thickness with calculated values for a square composition profile (dotted line) and a segregation profile with $E_{s}=0.34 \mathrm{eV}$ (solid line).

in good agreement with a previous secondary-ion mass spectroscopy (SIMS) measurement yielding $2.9 \mathrm{~nm}$ for $T_{\text {sub }}$ $=520^{\circ} \mathrm{C}^{15}$

Using the value of the chemical energy found for S1, the transition energies of $\mathrm{S} 2$ were calculated, fitting only the dependence of the In mole fraction in the wells on the absolute In source temperature, $T$. It was assumed that the flux rate of In atoms from the source has an exponential dependence on $T^{-1}$ in agreement with the vapor pressure dependence on the temperature, given by the Clausius-Clapeyron relation. In Fig. 2 the PL spectrum of S2 at $10 \mathrm{~K}$ is shown, and the measured transition energies are compared to the calculated values. For this fit, a maximum deviation of 2 $\mathrm{meV}$ for all six quantum wells was found. The difference between the nominal and the real In source calibration causes a change in the thickness of the wells, which has been taken

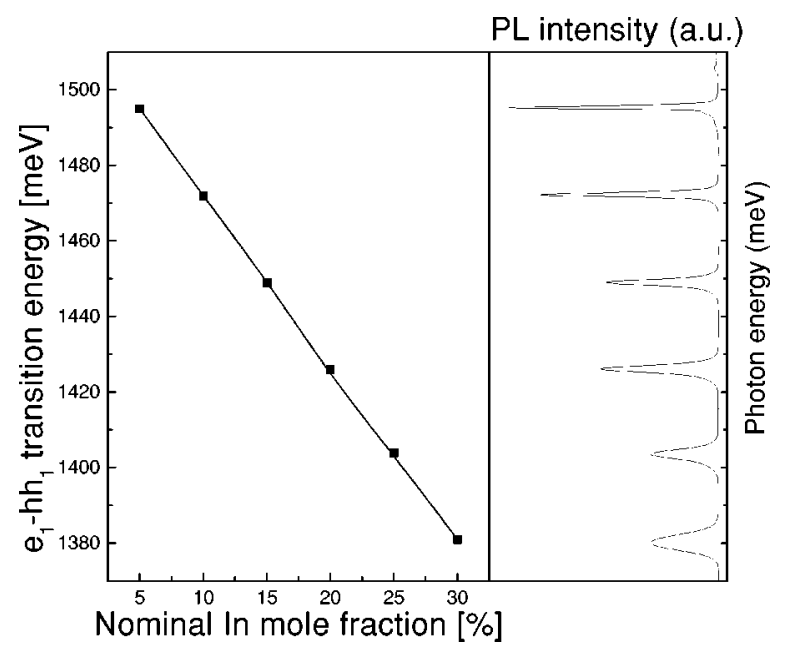

FIG. 2. Right: Photoluminescence spectrum of S2 at $T=10 \mathrm{~K}$. Left: Comparison of measured transition energies (squares) vs the nominal In mole fraction with calculated values for the best fit of the In source flux rates (solid line).

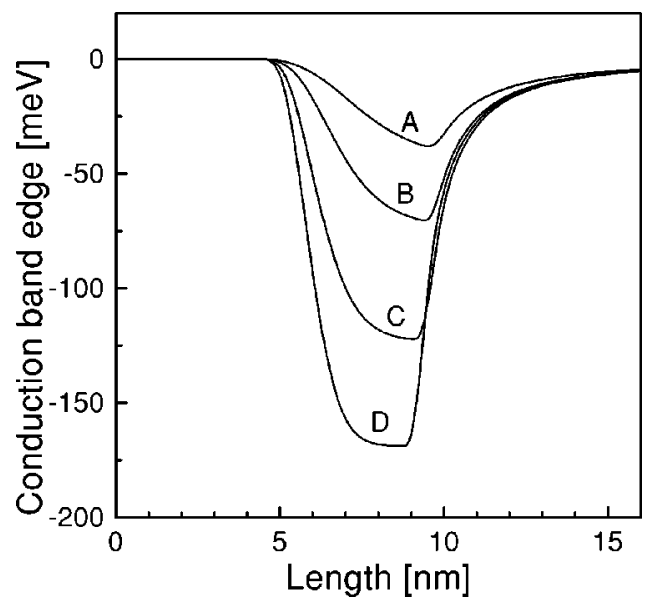

FIG. 3. The shape of the conduction band potential of four $\mathrm{InGaAs} / \mathrm{GaAs}$ quantum wells in S2, with a nominal thickness of $5 \mathrm{~nm}$ and nominal (real) In contents of A: $5 \%(5.5 \%)$, B: $10 \%(9.2 \%)$, C: $20 \%(15.6 \%)$ and D: $30 \%$ $(21.7 \%)$. The origin of the energy scale corresponds to the conduction band edge in GaAs.

into account in all the calculations described here. The shapes of the potentials in the quantum wells in S2 are shown in Fig. 3, represented by the energy of the conduction band edge. The deviation from an ideal square well potential is clear; however, for high In contents the first interface becomes more abrupt. This is due to the strain contribution to the reaction energy, ${ }^{14}$ which tends to inhibit In segregation for high In contents. The growths of S1 and S2 can be compared using the PL peak position of the $5 \mathrm{~nm}$ well with a nominal In content of 0.10 included in both samples. Their transition energies differ by less than $0.2 \mathrm{meV}$, indicating identical growth conditions and assuring the comparability of all three samples investigated here. Furthermore, the sharp PL lines of S1 and S2 indicate a good crystal quality and that the critical thickness has not been exceeded.

In Fig. 4 the PL spectrum of S3 at $T=10 K$ is shown. The In contents and widths of the wells according to the

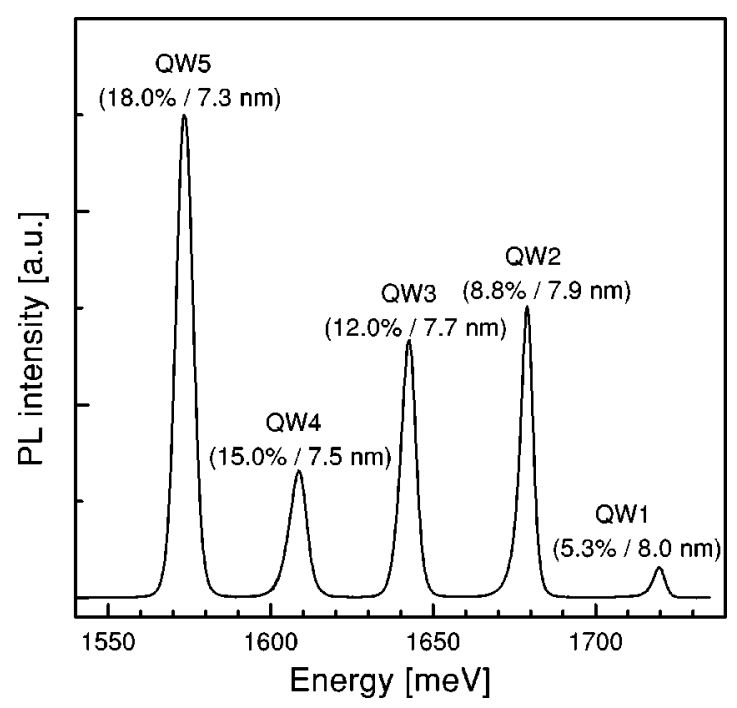

FIG. 4. Photoluminescence spectrum of $\mathrm{S} 3$ at $T=10 \mathrm{~K}$. The In content in the wells obtained by calibrating the In source with the PL peaks in S2, are stated in parenthesis. 


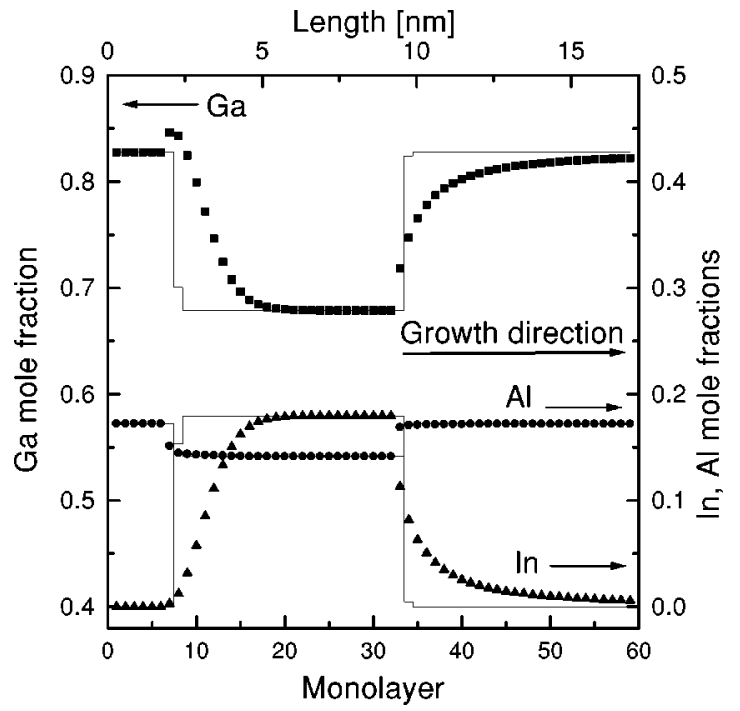

FIG. 5. Simulated composition profile of a 8-nm-thick quantum well with an In mole fraction of 0.18 (QW5 in S3), and $\mathrm{Al}_{0.17} \mathrm{Ga}_{0.83}$ As barriers. The calculated mole fractions of the group III elements are indicated with symbols (Ga: squares, $\mathrm{Al}$ : circles, In: triangles) and the composition profiles without segregation are shown with straight lines. Right axis: Al and In mole fractions, and left axis: Ga mole fraction.

calibration using the S2 PL-peak positions are indicated. The PL intensities of the quantum wells cannot be compared directly, since different excitation efficiencies and reabsorption effects in the structure should be considered. However, the PL-line shapes are identical; only the widths are different as will be addressed in Sec. V.

In order to model the composition profile of the quaternary quantum wells as described in Sec. III, knowledge of the chemical energy for each of the three reactions (1)-(3) is needed. Based on the value found from S1 we have used $E_{s}=0.17 \mathrm{eV}$ for $\mathrm{Ga}$ segregation in $\mathrm{AlGaAs}$ and $E_{s}$ $=0.52 \mathrm{eV}$ for In segregation in InAlAs, according to the experimentally determined ratio of approximately $1: 2: 3$ between these quantities. ${ }^{16,17}$ The simulated composition profile for QW5 is shown in Fig. 5. Since Al is the nonsegregating element in the quaternary InAlGaAs system, the transients in the In mole fraction at the interfaces of the quantum well are accompanied by a change in the Ga mole fraction, whereas the Al mole fraction is almost constant. Before the growth of the InAlGaAs layer, the surface is Ga rich due to Ga segregation in the AlGaAs barrier. However, in the quaternary well material the segregation of In is strongest, and hence the Ga rich surface is "pushed" into the bulk giving rise to the Ga peak at the first interface in Fig. 5. This peak is also reflected in the shape of the potential energy in the wells, shown in Fig. 6, where the shoulder at the first interface is due to the increased $\mathrm{Ga}$ mole fraction compared to the barrier region.

Fitting the calculated transition energies to the measured values, allows us to obtain an empirical band gap relation for unstrained $\operatorname{In}_{x}\left(\mathrm{Al}_{y} \mathrm{Ga}_{1-y}\right)_{1-x} \mathrm{As}$, in the form of a second order expansion for low values of $x$ and $y$. For this expansion the terms proportional to $x, y, x^{2}$, and $y^{2}$ are given by Eqs. (4) and (5), leaving only the term proportional to $x y$ to be determined. The fit then yields:

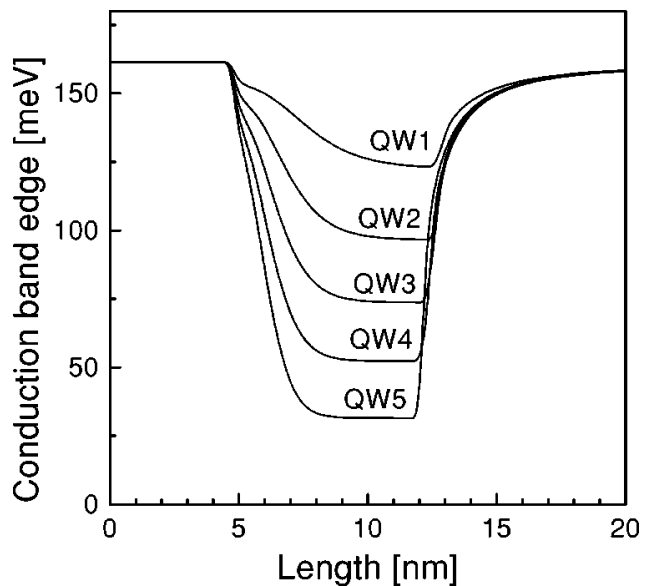

FIG. 6. The shape of the conduction band potential of the five $8 \mathrm{~nm}$ InAlGaAs/AlGaAs quantum wells in S3. The origin of the energy scale corresponds to the conduction band edge in GaAs.

$$
1.519+1.36 y-1.584 x+0.55 x y+0.22 y^{2}+0.475 x^{2} \text {. }
$$

The agreement between the measured e-hh and e-lh (measured with photoluminescence excitation spectroscopy) transition energies and the calculated values are shown in Fig. 7 and Table II. For the heavy holes the difference is less than $4 \mathrm{meV}$ for all the wells, whereas for the light holes it is less than $5 \mathrm{meV}$ for QW3-QW5 and $8 \mathrm{meV}$ for QW1 and QW2. Since the valence band potential is very shallow for QW1 and QW2, the light-hole wave functions penetrate into the barrier. Hence, the enhancement of the exciton binding energy due to quantum confinement is overestimated here, partly explaining the difference.

Since the empirical band gap relation is based on the well established relations for the ternary compounds InGaAs and AlGaAs, the term proportional to $x y$ could in principle have been determined by fitting the transition energy of a single InAlGaAs quantum well. However, the good agreement obtained for all the quantum wells in S3 where the In content is varied, further supports the band gap relation and the model for the In segregation used here.

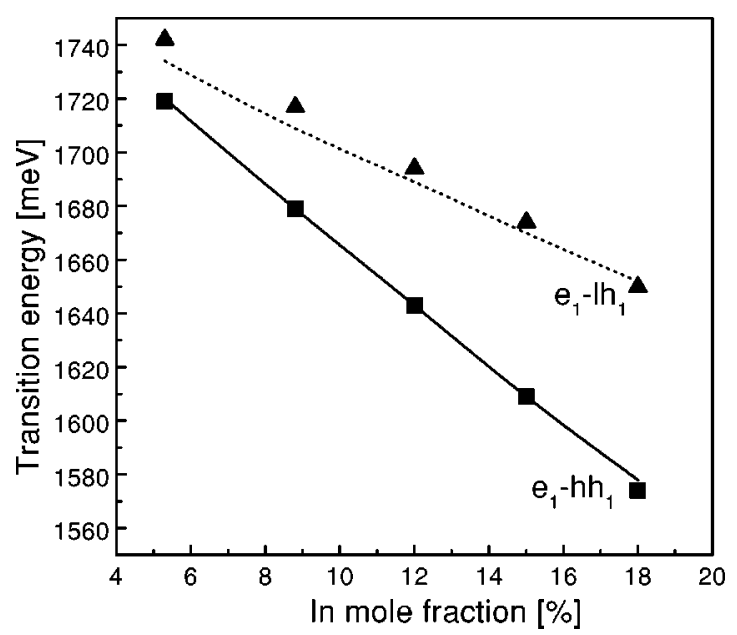

FIG. 7. Measured $e_{1}-h h_{1}$ (squares) and $e_{1}-l h_{1}$ (triangles) transition energies of S3, compared to calculated values for the heavy (solid line) and light hole (dotted line) using Eq. (6) for the band gap energy. 
TABLE II. Calculated and measured values for the $e$-hh and $e$-lh transitions and PL linewidths of S3. $L$ and $x$ denote the values of well thickness and $\ln$ content used in the calculations.

\begin{tabular}{|c|c|c|c|c|c|c|c|c|}
\hline & \multirow[b]{2}{*}{$L(\mathrm{~nm})$} & \multirow[b]{2}{*}{$x$} & \multicolumn{2}{|c|}{$E_{e-\mathrm{hh}}(\mathrm{eV})$} & \multicolumn{2}{|c|}{$E_{e-\mathrm{lh}}(\mathrm{eV})$} & \multicolumn{2}{|c|}{ FWHM (meV) } \\
\hline & & & meas. & calc. & meas. & calc. & meas. & calc. \\
\hline QW1 & 8.0 & 0.052 & 1.719 & 1.720 & 1.742 & 1.734 & 3.5 & 3.50 \\
\hline QW2 & 7.9 & 0.088 & 1.679 & 1.679 & 1.717 & 1.709 & 3.9 & 3.93 \\
\hline QW3 & 7.7 & 0.12 & 1.643 & 1.643 & 1.694 & 1.689 & 4.6 & 4.62 \\
\hline QW4 & 7.5 & 0.15 & 1.609 & 1.609 & 1.674 & 1.670 & 5.4 & 5.32 \\
\hline QW5 & 7.3 & 0.18 & 1.574 & 1.578 & 1.650 & 1.652 & 6.0 & 5.99 \\
\hline
\end{tabular}

\section{LINEWIDTH ANALYSIS}

In Table II, the measured linewidths, full width at half maximum (FWHM), of the five InAlGaAs quantum wells are compared to the linewidths expected from random alloy fluctuations (RAF) and well width fluctuations (WWF). Based on a previous analysis for ternary bulk semiconductors, ${ }^{26}$ the following relation was used to calculate the FWHM broadening due to the random distribution of $\mathrm{In}$ in a $\operatorname{In}_{x}\left(\mathrm{Al}_{y} \mathrm{Ga}_{1-y}\right)_{1-x} \mathrm{As}$ quantum well:

$$
\Delta E_{x}=2 \sqrt{2 \ln 2} \sqrt{\frac{x(1-x)}{N}} \frac{\partial E_{\text {gap }}}{\partial x} .
$$

The same relation was used for the $\mathrm{Al}$ distribution. $N$ is the number of atoms contained in the volume of the exciton, $V$. Here, we have used $V=\pi a_{0}^{2} L_{z}$, where $a_{0}$ is the in plane exciton Bohr radius and $L_{z}$ is the FWHM size of the electron wave function in the direction of growth. However, for QW1 and QW2, $L_{z}$ was set to the well width, because the FWHM size is larger than the well width. The best fit was found for $a_{0}=13 \mathrm{~nm}$, which is smaller than the exciton Bohr radius in GaAs, due to the enhanced exciton binding energy in a quantum well compared to a bulk structure. The FWHM broadening due to WWF is given by:

$$
\Delta E_{\mathrm{WWF}}=\frac{\partial E_{e-h h}}{\partial L} \Delta L
$$

where $L$ is the thickness of the well and $\Delta L$ is an effective interface roughness. The derivative of the transition energy with respect to the thickness was calculated for the quantum wells individually and for the effective roughness a value of 1.1 ML was obtained, which is comparable to typical values obtained for AlGaAs structures. Assuming that the three contributions to the broadening are independent, the total FWHM broadening is given by:

$$
\Delta E_{\mathrm{tot}}=\sqrt{\left(\Delta E_{x}\right)^{2}+\left(\Delta E_{y}\right)^{2}+\left(\Delta E_{\mathrm{WWF}}\right)^{2}} .
$$

For QW1-QW5, $E_{\mathrm{WWF}}$ varies almost linearly with the In content from 0.77 to $3.85 \mathrm{meV}$, whereas $E_{x}\left(E_{y}\right)$ varies sublinearly from $1.75(2.93 \mathrm{meV})$ to $3.19 \mathrm{meV}(3.29 \mathrm{meV})$. Since the dependencies of WWF and RAF on the In content in QW1-QW5 are different, the two broadening mechanisms can be distinguished in the measured line widths and hence the parameters in the model, $a_{0}$ and $\Delta L$, are well determined.
Due to the good agreement between the measured and calculated linewidths, we conclude that the distribution of group III atoms in the InAlGaAs quantum wells does not show any signs of clustering, and is well described by random statistics.

\section{CONCLUSION}

The transition energies and linewidths of several InAlGaAs/AlGaAs quantum wells grown on a GaAs substrate, have been measured and modeled. Using two reference samples with InGaAs/GaAs quantum wells grown under the same conditions, it was possible to make a calibration of the In growth rate and the chemical energies of the In segregation. A good fit to the $e$-hh and $e$-lh transitions was found using a band gap relation for $\operatorname{In}_{x}\left(\mathrm{Al}_{y} \mathrm{Ga}_{1-y}\right)_{1-x} \mathrm{As}$, Eq. (6), based on the well known relations for $\operatorname{In}_{x} \mathrm{Ga}_{1-x} \mathrm{As}$ and $\mathrm{Al}_{y} \mathrm{Ga}_{1-y} \mathrm{As}$, and adding a term $0.55 x y$ to account for the simultaneous presence of In and Al. From the linewidths, an effective interface roughness of 1.1 ML was found, using a model that includes well width fluctuations and random alloy fluctuations. Hence, the linewidths do not show indications of clustering or dislocation formation in the InAlGaAs/ AlGaAs structures.

\section{ACKNOWLEDGMENTS}

The authors would like to thank C. B. Sørensen at the III-V Nanolab for helpful discussions on the growth of the samples. This work was supported by the Danish Natural Science Research Council.

${ }^{1}$ D. Olego, T. Y. Chang, E. Silberg, E. A. Caridi, and A. Pinczuk, Appl. Phys. Lett. 41, 476 (1982).

${ }^{2}$ J. C. Fan and Y. F. Chen, J. Appl. Phys. 80, 1239 (1996).

${ }^{3}$ Y. F. Chen, Y. T. Dai, J. C. Fan, T. L. Lee, and H. H. Lin, Appl. Phys. Lett. 67, 1256 (1995).

${ }^{4}$ H. Hillmer, R. Losch, and W. Schlapp, J. Appl. Phys. 77, 5440 (1995).

${ }^{5}$ R. P. Leavitt and J. L. Bradshaw, J. Appl. Phys. 76, 3429 (1994).

${ }^{6}$ L. A. Cury, J. Beerens, and J. P. Praseuth, Appl. Phys. Lett. 63, 1804 (1993).

${ }^{7}$ R. F. Kopf, H. P. Wei, A. P. Perley, and G. Livescu, Appl. Phys. Lett. 60, 2386 (1992)

${ }^{8}$ Y. Hirayama, W. Choi, L. H. Peng, and C. G. Fonstad, J. Appl. Phys. 74, 570 (1993).

${ }^{9}$ J. Ko, C.-H. Chen, and L. A. Coldren, Electron. Lett. 32, 2099 (1996).

${ }^{10}$ S. Bhargava, C. Zheng, J. Ko, M. A. Chin, L. A. Coldren, and V. Narayanamurti, Appl. Phys. Lett. 73, 3271 (1998).

${ }^{11}$ R. Nötzel, Semicond. Sci. Technol. 11, 1365 (1996).

${ }^{12}$ Properties of Lattice-matched and Strained Indium Gallium Arsenide, edited by P. Bhattacharya (INSPEC, London, 1993).

${ }^{13}$ T. G. Andersson, Z. G. Chen, V. D. Kulakovskii, A. Uddin, and J. T. Vallin, Appl. Phys. Lett. 51, 752 (1987).

${ }^{14}$ J. M. Moison, C. Guille, F. Houzay, F. Barthe, and M. V. Rompay, Phys. Rev. B 40, 6149 (1989)

${ }^{15}$ K. Muraki, S. Fukatsu, Y. Shiraki, and R. Ito, Appl. Phys. Lett. 61, 557 (1992)

${ }^{16}$ J. M. Gerard and J. Y. Marzin, Phys. Rev. B 45, 6313 (1992).

${ }^{17}$ J. M. Gerard, Appl. Phys. Lett. 61, 2096 (1992).

${ }^{18}$ P. Y. Yu and M. Cardona, Fundamentals of Semiconductors (Springer, Berlin, 1996).

${ }^{19}$ R. Atanasov, F. Bassani, A. D’Andrea, and N. Tomassini, Phys. Rev. B 50, 14381 (1994).

${ }^{20}$ L. Pavesi and M. Guzzi, J. Appl. Phys. 75, 4779 (1994).

${ }^{21}$ Properties of Aluminium Gallium Arsenide, edited by S. Adachi (INSPEC, London, 1993).

${ }^{22}$ Numerical Data and Functional Relationships in Science and Technology, 
Volume 17a, Semiconductors, edited by O. Madelung, M. Schulz, and H. Weiss (Springer, Berlin, 1982).

${ }^{23}$ Physical Properties of III-V Semiconductor Compounds, edited by S. Adachi (Wiley, New York, 1992).
${ }^{24}$ C. G. Van de Walle, Phys. Rev. B 39, 1871 (1989).

${ }^{25}$ N. Grandjean, J. Massies, and M. Leroux, Phys. Rev. B 53, 998 (1996).

${ }^{26}$ E. Schubert, E. O. Göbel, Y. Horikoshi, K. Ploog, and H. J. Queisser, Phys. Rev. B 30, 813 (1984). 